\title{
Study on tension variation characteristics of hoisting wire rope of high speed traction elevator
}

\author{
Qing Zhang \\ School of Mechanical and Electrical Engineering \\ Shandong Jianzhu University \\ Jinan, China \\ zhangqing@sdjzu.edu.cn \\ Zhe Yang \\ School of Mechanical and Electrical Engineering \\ Shandong Jianzhu University \\ Jinan, China \\ 1334664718@163.com
}

\author{
Tao Hou \\ School of Mechanical and Electrical Engineering \\ Shandong Jianzhu University \\ Jinan, China \\ sddpchen@163.com \\ Ruijun Zhang \\ School of Mechanical and Electrical Engineering \\ Shandong Jianzhu University \\ Jinan, China \\ Zhangruijun@sdjzu.edu.cn
}

\begin{abstract}
For the study on tension variation characteristics of hoisting wire rope of high speed traction elevator, the hoisting wire rope time-varying model is built in the process of high speed traction elevator ascending and descending, the ideal run curve of elevator ascending and descending is obtained by fitting the quintic polynomial, also served as the input parameter of the time-varying model parameters of the hoisting wire rope tension. Research shows that the hoisting wire rope tension will reach the maximum when the elevator runs evenly. Changes in elevator acceleration can cause a sudden change in tension of the hoisting wire rope.
\end{abstract}

Keywords-High speed elevator; lifting wire rope; tension; changing rule

\section{INTRODUCTION}

The elevator is called the "the vertically moving car" in the building space, with the number of high-rise building and super high-rise building constantly increasing, the elevator gradually develops towards large travel and high speed.

Hoisting wire rope as the traction and bearing component of high speed elevator, twisted by high strength steel wire, own the advantages of high tensile strength, strong dynamic load overload capacity and so on, meanwhile, due to the characteristics of large travel and high speed of high speed elevator, the tension unbalance of hoisting wire rope and the uncertainty of working load and environment, serious problems of fatigue, corrosion and wear will occur in the working process of hoisting wire rope, seriously affect the safety of high-speed elevators [1]. At present, domestic scholars have done plenty of research work in the dynamic characteristics of high-speed elevators and various stochastic uncertainty problem [2-6], but insufficient for the tension of hoisting wire rope $[7,8]$, the research on the change of the tension of the hoisting wire rope in the process of elevator up and down has not been studied yet. Therefore, it is necessary to

1 The Natural Science Foundation of Shandong Province, Project number: ZR2017MEE049

2 Introduce urgently needed talents project for the western economic uplift belt and the key areas of poverty alleviation and development in Shandong Province study the change rule of the tension of the hoisting wire rope during the operation of the high speed elevator.

When the elevator running, the length of the hoisting wire rope increases or shortens with the operation of the elevator, has a certain time varying characteristic. The general numerical analysis method is difficult to achieve the purpose of high precision for the tension control equation of high-speed elevator hoisting wire rope constructed in this paper. It is necessary to adopt a numerical calculation method with high computational accuracy and explicit absolute stability to enhance the simulation analysis of the tension of the hoisting wire rope.

Considering these problems, the time - varying model of hoisting wire rope tension was established, the precise integration method is used to simulate it, and which reveals the rule of the tension of the hoisting wire rope during the ascending and descending process of the high-speed elevator.

\section{ESTABLISHMENT OF TIME VARYING MODEL FOR HOISTING WiRE ROPE TENSION OF HIGH SPEED ELEVATOR}

The hoisting wire rope which stretched from the top of the high-speed elevator car to the traction wheel is used as the objector of study in this paper, considering the tension provided by the tensioning system, modeling the tension of hoisting wire rope of high speed elevator, in order to improve the efficiency of simulation analysis of the model built, the following assumptions are made:

(1) The elevator rope is uniform and continuous, the linear density, cross sectional area and elastic modulus of the wire rope remain unchanged during the operation of the elevator;

(2) Ignoring the airflow in the elevator hoistway and the influence of the creeping force of the guide shoe and guide rail; 
(3) Ignore the influence of elevator Transverse Excitation.

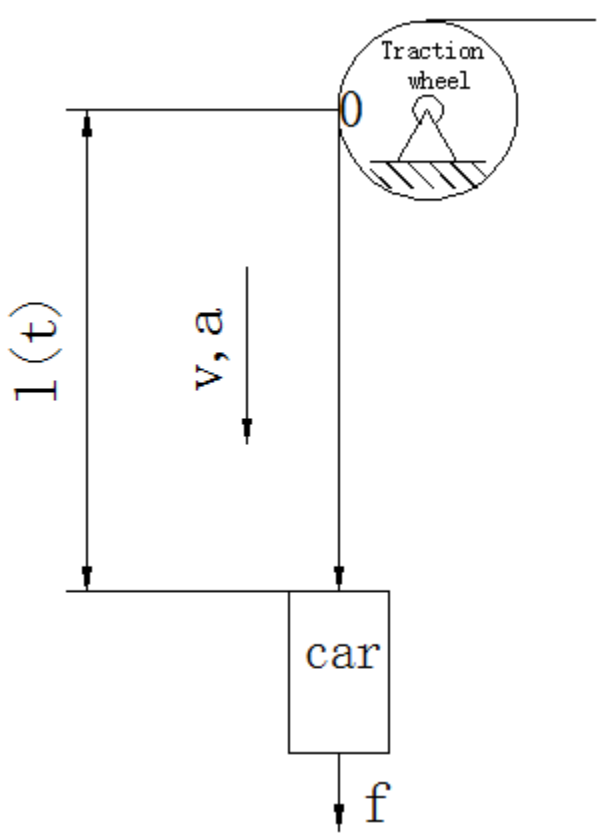

Fig. 1. Dynamic model of elevator motion.

Take the downward direction of elevator speed, acceleration as positive, when the elevator goes up, the tension control equation [9] is as follows

$$
\ddot{F}-\frac{2 v}{l(t)} \dot{F}+\frac{\left[\frac{E A}{m+m_{\text {car }}+f+\rho l(t) / 3}+a\right]}{l(t)} F=\frac{E A(g-a)}{l(t)}
$$

When the elevator goes down, the tension control equation is as follows:

$$
\ddot{F}+\frac{2 v}{l(t)} \dot{F}+\frac{\left[\frac{E A}{m+m_{\mathrm{car}}+f+\rho l(t) / 3}+a\right]}{l(t)} F=\frac{E A(g+a)}{l(t)}
$$

Where: $F$ - traction rope tension of high speed elevator; $E$ - elastic modulus of traction rope for elevator; A A sectional area of traction rope for elevator; $v$ - the running speed of elevator; $g$-gravitational acceleration, $9.8 \mathrm{~m} / \mathrm{s}^{2} ; \rho$ -linear density of elevator wire rope; $m 、 m_{\text {car }} 、 f$ - express the rated load quality of the elevator, the quality of the car and the tension of the tensioning system respectively; The tension control equation of the elevator hoisting wire rope can be expressed as a standard dynamic equation:

$$
M \ddot{\mathrm{F}}+C(t) \dot{F}+K(t) F=f(t)
$$

Among them:

$$
M=1
$$

$$
\begin{gathered}
C(t)= \pm \frac{2 v}{l(t)} \\
K(t)=\frac{\left[\frac{E A}{m+m_{\text {轿厢 }}+f+\rho l(t) / 3}+a\right]}{l(t)} \\
f(t)=\frac{E A(g \pm a)}{l(t)}
\end{gathered}
$$

\section{Precise Integration Method for Time VARYing} MODEl OF ElEVATOR HOISTING ROPE TENSION

Precise time integration method [10] is a kind of new numerical method put forward by Professor Zhong Wanxie, which has been widely used in the linear constant system [11], and produce good effects.

First, we introduce the dual variable by imitating the Hamiltonian system, make

$$
p=M \dot{\mathrm{F}}+C(t) F / 2 \text { or }
$$

$$
\dot{\mathrm{F}}=M^{-1} p-M^{-1} C(t) \mathrm{F} / 2
$$

By substituting the above formula into the equation, the following equation can be obtained

$$
\dot{p}=\left(C(t) M^{-1} C(t) / 4-K(t)\right) \mathrm{F}-C(t) M^{-1} p / 2+f(t)
$$

The above equations are written in the general form of a linear system

$$
\left\{\begin{array}{l}
\dot{\mathrm{q}}=A q+C p+r_{q} \\
\dot{\mathrm{p}}=B q+D p+r_{p}
\end{array}\right.
$$

Where: $q=F, p=p$

$$
\begin{gathered}
A=-M^{-1} C(t) / 2 \\
B=C(t) M^{-1} C(t) / 4-K(t) \\
C=-C(t) M^{-1} / 2 \\
D=M^{-1} \\
\mathrm{r}_{\mathrm{p}}=f(t), \mathrm{r}_{\mathrm{q}}=0
\end{gathered}
$$


Therefore,

$$
\dot{z}=H z+\phi(t)
$$

Where, $\mathrm{z}=\left[\begin{array}{c}\mathrm{q} \\ p\end{array}\right], \quad \mathrm{H}=\left[\begin{array}{ll}A & D \\ B & \mathrm{C}\end{array}\right], \quad \phi(t)=\left[\begin{array}{c}\mathrm{r}_{q} \\ r_{p}\end{array}\right]$

Given that the non-homogeneous term is linear in the time step $\left(t_{k}, t_{k+1}\right)$, the equation is

$$
\dot{z}=H z+\phi_{k}+\dot{\phi}_{k}\left(t-t_{k}\right)
$$

Then, the solution at $t_{k+1}$ moment can be written as

$$
\begin{aligned}
& z_{k+1}=T_{\mathrm{k}}\left[z_{k}+H_{k}^{-1}\left(\phi_{k}+H_{k}^{-1} \dot{\phi}_{k}\right)\right]-H_{k}^{-1}\left[\phi_{k}+\right. \\
& \left.H_{k}^{-1} \dot{\phi}_{k}+\dot{\phi}_{k}\left(t_{k+1}-t_{k}\right)\right]
\end{aligned}
$$

Where: $T_{\mathrm{k}}=e^{H_{k}\left(t_{k+1}-t_{k}\right)}$

According to (8), the steps were gradually performed to obtain $z_{1}, z_{2}, z_{3}, \cdots, z_{k}, \cdots$

\section{EXAMPLE ANALYSIS}

The simulation analysis of a high speed elevator lifting system is carried out, the tractive rope density is $\rho=0.87 \mathrm{~kg} / \mathrm{m}$, and sectional area is $A=89.344 \mathrm{~mm}^{2}$, the quality of car is $m_{\text {car }}=300 \mathrm{~kg}$, the rated load is $m=150 \mathrm{~kg}$, tension provided by tensioning system is $f=300 \mathrm{~N}$.

When the precise integration method is used to simulate the kinetic equation, need to input elevator operating parameters, the elevator operating curve is assumed to be ideal in this paper, the elevator completes an uplink or downlink process is divided into 7 stages, the running state of elevator in each phase lists in Table I, after giving the elevator maximum speed, $v_{\max }=5 \mathrm{~m} / \mathrm{s}$ maximum acceleration, Rising height, $l_{\max }=132.5 \mathrm{~m}$, jerk speed, $j_{\max }=1 \mathrm{~m} / \mathrm{s}^{3}$ and Running time of each stage (see Table I), by fitting quintic polynomial (9), we can get the elevator running curve of each stage.

$$
l_{i}(t)=C_{0}^{i}+C_{1}^{i} t+C_{2}^{i} t^{2}+C_{3}^{i} t^{3}+C_{4}^{i} t^{4}+C_{5}^{i} t^{5}
$$

By derivation, the expressions of the running speed, acceleration and jerk speed of the elevator can be obtained. Fig. 2 is the curve of the running state of the high speed elevator in the process of up and down.
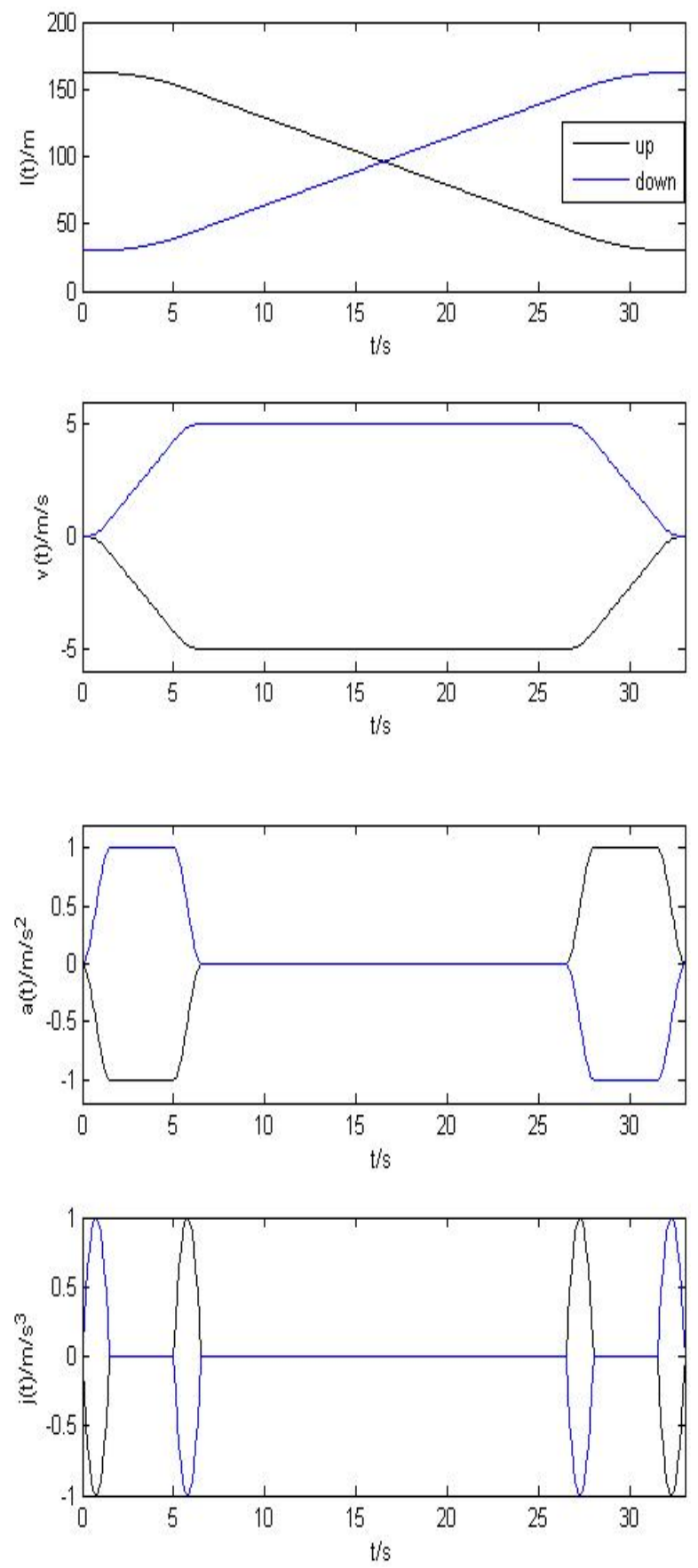

Fig. 2. Movement profile of elevator

As Fig. 3 shows, it's the tension curve of hoisting wire rope during elevator up, in combination with Table I, the tension curve of hoisting wire rope is analyzed in Fig. 3, when the elevator up, the length of the wire rope is gradually shortened when the elevator goes up, there are sudden changes in tension of hoisting wire rope when the elevator running 1,3 , 5 , 7 stage, it can be inferred that the sudden change in tension of the hoisting wire rope is related to the change in acceleration, when the elevator up uniformly, the tension of the hoisting rope has been evenly reduced in general, it can be 
deduced that this change is related to the reduction in the length of the hoisting wire rope, the maximum tension of the wire rope is raised in the second stage of the elevator upstream process, the maximum tension is $10245 \mathrm{~N}$.

TABLE I. REGION OF ELEVATOR DOWNWARD MOVEMENT PROFILE.

\begin{tabular}{ccc}
\hline Stage & Time required & Stage description \\
\hline 1 & $t_{j}$ & Acceleration increases to $a=a_{\max }$ \\
2 & $t_{a}$ & $\begin{array}{c}\text { Acceleration remains } a_{\max } \text { constant } \\
\text { The acceleration is reduced to zero, }\end{array}$ \\
3 & $t_{j}$ & $v=v_{\max }$ \\
4 & $t_{v}$ & Keep the speed $v_{\text {max }}$ \\
$t_{j}$ & Acceleration increases to $a=-a_{\max }$ \\
6 & $t_{a}$ & Acceleration remains $-a_{\max }$ constant \\
7 & $t_{j}$ & The acceleration is reduced to zero, \\
& & $v=0$
\end{tabular}

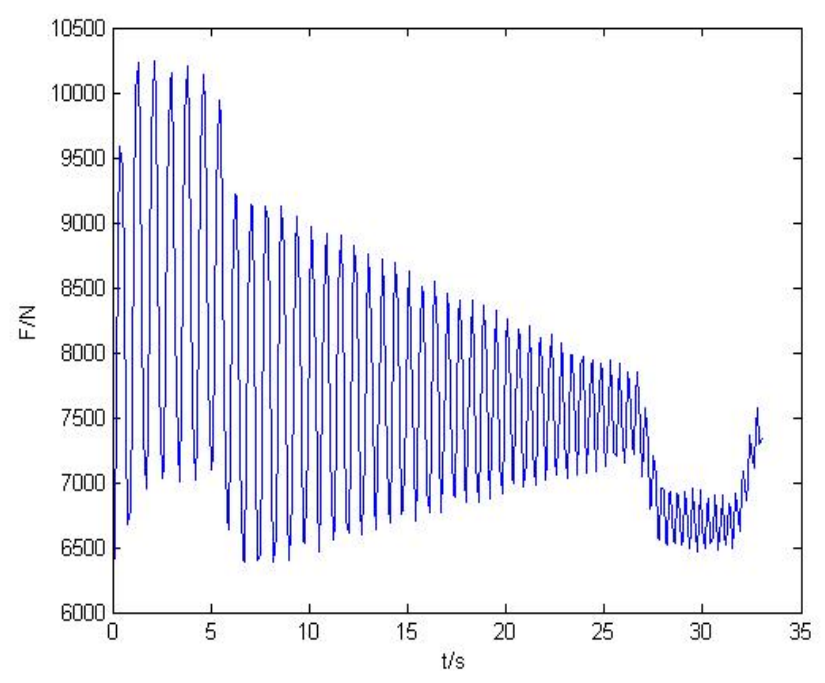

Fig. 3. Tension curve of hoisting wire rope during elevator up

As Fig. 4 shows, it's the tension curve of hoisting wire rope during elevator down, associated with Table I, analyzing the tension curve of hoisting wire rope in Fig. 4, the length of the hoisting wire rope is increasing as the elevator descends, also sudden changes in tension of hoisting wire rope with the change of elevator running acceleration, at elevator uniform speed downstream stage, the tension is not much change, the maximum tension of the wire rope is raised in the second stage of the elevator downstream process, the maximum tension is $10618 \mathrm{~N}$.

It's not difficult find that, combined with the tension curve of the hoisting wire rope during elevator up and down, whether the elevator up or down, the maximum tension of the hoisting wire rope appears in the uniform acceleration phase when the elevator is running.

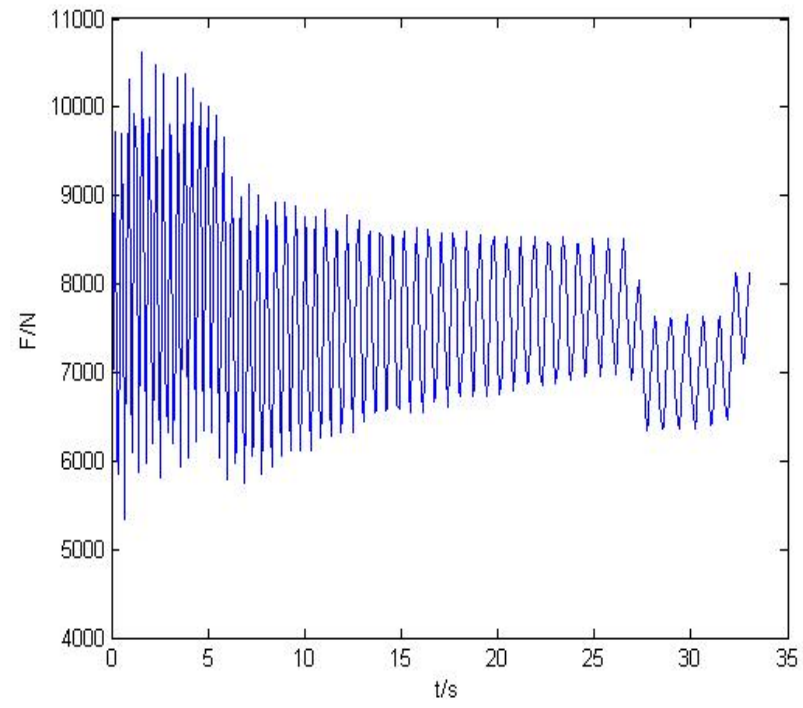

Fig. 4. Tension curve of hoisting wire rope during elevator up

\section{CONCLUSION}

(1) The ideal running state of elevator, which is fitted by using the quintic polynomial in this paper, and input as the parameter of time-varying model simulation for tension of hoisting wire rope in high speed elevator, solved by the precise integration method of elevator wire rope tension time-varying model, eventually obtained the tension of each time of the hoisting wire rope by simulation. Research shows: the elevator hoisting wire rope tension is maximum when the elevator runs evenly, the change of acceleration will lead to a huge change of elevator rope tension.

(2) In this paper, the dynamic tension characteristics of high speed traction elevator hoisting wire rope are studied, which has important guiding significance for the improvement of high speed elevator lifting strategy and the selection of wire rope.

\section{REFERENCE}

[1] P. Pavel and K. Jozef. "Failure analysis of hoisting steel wire rope”. Eng Fail. Anal. vol. 45, pp. 96-105, 2014.

[2] J.H. Bao, P. Zhang, C.M. Zhu. "Modeling and control of longitudinal vibration on flexible hoisting systems with time-varying length". Sci. Verse. Sci. Dir. vol. 15, pp. 4521-4526, 2011.

[3] C. Wang, R.J. Zhang and Q. Zhang. “Analysis of Transverse Vibration Acceleration for a High-speed Elevator with Random Parameter Based on Perturbation Theory”. Int. J. Acoust. Vibr. vol. 22 no. 2, pp. 218223, 2017.

[4] X.Q. Du, D.Q. Mei and Z.C. Chen. "Time-varying model and horizontal vibration response analysis of high speed traction elevator”. J. Zhejiang Univ. vol. 43, no. 1, pp. 148-152, 2009.

[5] J.H. Bao, P. Zhang, C.M. Zhu. "Longitudinal vibration characteristics of wire rope with variable length hoisting system”. Vibr. Shock, vol. 32 no. 15, pp. 173-177, 2013.

[6] H. Wu, W.H. Ye, Y. Shen and Z.R. Tang. "Modeling and experimental analysis of vertical vibration of high speed elevator”, Mach. Manuf. vol. 51, no. 584, pp. 19-22, 2013.

[7] Y.L. Pan and X. Shi. "Finite element analysis of dynamic characteristics of wire rope traction transmission”. Mech. Des. Res. vol. 33, no. 4, pp. 152-154, 2017. 
[8] S.D. Yin, R.H. Qiu and Z.Q. Wang. "Design and implementation of tension monitoring system for elevator wire rope”. Microcomput. Inform. vol. 27, no. 6, pp. 33-35, 2011.

[9] P. Wang, X.M. Xiao, B.H. Ding and S.B. Li. "Dynamic simulation of hoisting wire rope in hoisting process”. Hoist. Conv. Mach. vol. 7, pp. 84-87, 2009.

[10] W.X. Zhong. "Precise integration method for transient process". Comput. Struct. Mech. Its Appl. vol. 12 no. 1, pp. 1-6, 1995.

[11] Z.C. Deng, H.J. Zheng and Y.L. Zhao, "Calculation of dynamic characteristics of extended cantilever structures based on precise integration method”. Aerosp. J. vol. 22 no. 6, pp. 110- 113, 2001. 